\title{
Analysis and Control of a Dissipative Spring-Mass Hopper with Torque Actuation
}

\author{
M. Mert Ankarali \\ Dept. of Electrical and Electronics Eng., \\ Middle East Technical University, \\ 06531 Ankara, Turkey \\ ankaralideee.metu.edu.tr
}

\author{
Uluç Saranlı \\ Dept. of Computer Engineering, \\ Bilkent University, \\ 06800 Ankara, Turkey \\ saranli@cs.bilkent.edu.tr
}

\begin{abstract}
It has long been established that simple springmass models can accurately represent the dynamics of legged locomotion. Existing work in this domain, however, almost exclusively focuses on the idealized Spring-Loaded Inverted Pendulum (SLIP) model and neglects passive dissipative effects unavoidable in any physical robot or animal. In this paper, we extend on a recently proposed analytic approximation to the stance trajectories of a dissipative SLIP model to analyze stability properties of a planar hopper with a single rotary actuator at the hip. We first describe how a suitably chosen torque controller can compensate for damping losses, maintaining the same energy level across strides and hence reducing the return map to a single dimension. We then identify and characterize equilibrium points for this return map under a fixed leg placement policy and show that "uncontrolled" asymptotic stability is feasible for this energy-regulated system. Subsequent presentation of simulation evidence establishes that the predictions of this approximate model are consistent with the exact plant model. The paper concludes with the application of our energy-regulation scheme to the design of a task-level gait controller that uses explicit leg placement commands in conjunction with the hip torque.
\end{abstract}

\section{INTRODUCTION}

Long term practical utility of mobile robots in unstructured environments critically depends on their locomotory aptitude. In this context, the performance of ground mobility that can ultimately be achieved by legged platforms is superior to any other alternative as evidenced by numerous examples in nature as well as a number of very successful dynamically stable autonomous legged robots that have been built to date $[10,25,26,30,37]$. Unfortunately, even on flat ground, legged morphologies do not enjoy the simplicity of models supported by the conveniently constrained and continuous modes of ground interaction observed in wheeled and, to some extent, tracked vehicles. Even the most basic legged behaviors such as walking and running require hybrid dynamic models whose analysis and control involve difficult challenges [14, 20, 23]. In the world of quasi-static locomotion with multi-legged robots, one can recover some of this simplicity through active or structural suppression of second order dynamics [39], but these methods are not directly applicable to dynamically dexterous modes of locomotion such as running.

One of the most significant discoveries in this context was most likely the recognition of similar center of mass (COM) movement patterns in running animals of widely different sizes and morphologies $[1,6,7,9,24]$. This led to the development of the simple yet accurate Spring-Loaded Inverted Pendulum (SLIP) model to describe such behaviors [21,34]. Significant research effort was devoted to both the use of this model as a basis for the design of fast and efficient legged robots [10, 19, 27,30 ] as well as its analysis to reveal fundamental aspects of associated locomotory behaviors [20]. The present paper falls into the latter category and contributes by investigating the previously unaddressed question of how the presence of passive damping affects the behavioral characteristics of running with the SLIP model.

Our treatment of this question is based on the use of analytic approximations to the otherwise non-integrable stance dynamics of the model. A number of such approximations have already been proposed in the literature. In particular, [35] uses a Hamiltonian formulation of the SLIP dynamics with an iterative application of the mean-value theorem to obtain an accurate return map for symmetric steps. More recently, [16] presents an analytically simple approximation to the stance dynamics of a conservative SLIP equipped with a linear spring. This approach is based on a linearization of gravity around mid-stance, similar in form to the solution proposed in [28] but involving a much more carefully formulated derivation. Inaccuracies of these approximations in the presence of nonsymmetric gravitational effects were partially addressed in [5] using explicit corrections. In contrast to the lossless models adopted in all these approaches, a new return map for the lossy SLIP model with viscous damping in the leg was proposed in [4], providing a basis for our present inquiry.

Despite the availability of methods to analyze stability properties of locomotory behaviors in the absence of closed-form expressions for a Poincaré map [2,3], a number of different possible approaches become available once a sufficiently accurate analytic return map is available. For example, [18] investigates in depth stability properties of a SLIP model attached to a rigid body by neglecting the effects of gravity, which allows for the derivation of suitable closed-form expressions for stride trajectories. A similar but less rigorous stability analysis was provided in [16] with comparisons to previous numerical results in [36] as well as biological data. In contrast to the lack of feedback control in these characterizations, [33] studies the stability of the SLIP model under a novel leg placement 
control strategy that only relies on easily obtainable state measurements. Another inquiry on how to achieve effective control of the SLIP model, now extended to a spatial setting, is provided in [8], focusing on lateral motions of the model. In conjunction with these studies primarily focused on running behaviors, similar analytically motivated contributions were also made to the structurally different walking behaviors [15, $22,40]$, providing evidence that the same dynamic model can provide a unified description for both walking and running [17].

Our contributions in the present paper have a number of important differences from existing work. Firstly, our plant model is dissipative, impairing the accuracy of most existing analytic approximations and associated predictions. Secondly, in contrast to the usual energy regulation mechanisms in the literature through adjustments of the leg length or changing stiffness, our model uses only a single torque actuator at the hip relative to a virtual body with fixed orientation to compensate for energy losses. These changes are motivated by being much more realistic from an implementation point of view, as evidenced by the successful use of similar actuation mechanisms in the Scout quadrupeds [26] and the RHex hexapod [30] as well as a number of other monopedal platforms $[12,32]$. Finally, our approximate solutions to the return map also take into account the effect of gravity on the angular momentum for steps that are non-symmetric with respect to the gravitational vertical, increasing the practical applicability of associated stability results.

\section{The Torque-Actuated Dissipative SLIP Model}

\section{A. System Dynamics and the Apex Return Map}

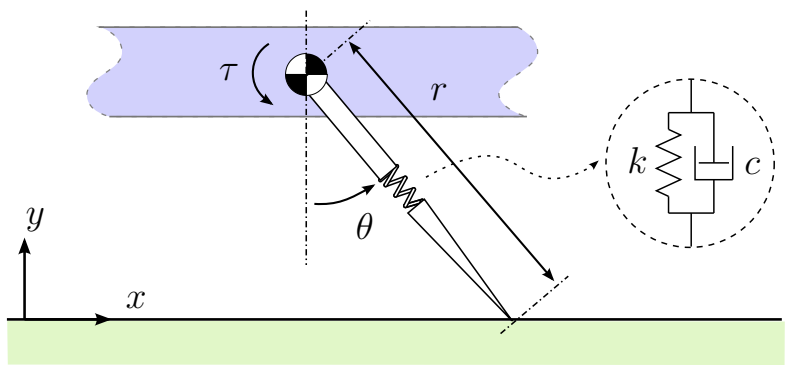

Fig. 1. TD-SLIP : Dissipative spring-mass hopper with rotary hip actuation

Fig. 1 illustrates the Torque-actuated Dissipative SpringLoaded Inverted Pendulum (TD-SLIP) plant we investigate in this paper. It consists of a fixed orientation (2-DOF) planar rigid body with mass $m$, connected to a massless, fully passive leg with linear compliance $k$, rest length $r_{0}$ and linear viscous damping $c$, through an actuated rotary joint with torque $\tau$. The system alternates between stance and flight phases during running, with the flight phase further divided into the ascent and descent subphases. Fig. 2 illustrates the three important events that define transitions between these phases: touchdown, where the leg comes into contact with the ground, liftoff, where the toe takes off from the ground and finally apex, where the body reaches its maximum height during flight with $\dot{y}=0$. Another important event, not illustrated in the figure, is bottom, corresponding to the point of maximal leg compression during stance. Table I details the notation used throughout the paper.

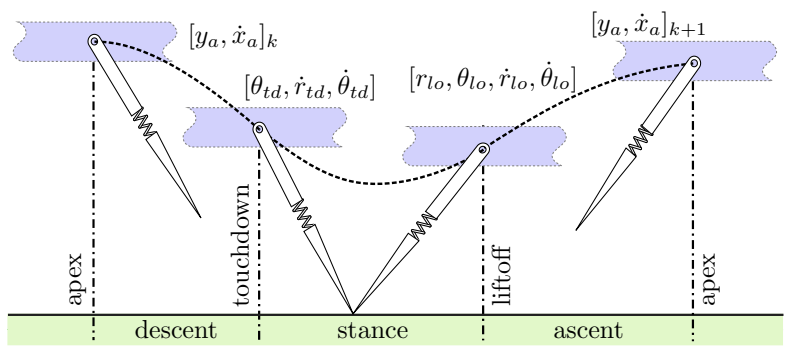

Fig. 2. A single TD-SLIP stride with definitions of transition states

TABLE I

NOTATION USED THROUGHOUT THE PAPER

\begin{tabular}{|c|l|}
\hline \multicolumn{2}{|c|}{ System States, Event States and Control Inputs } \\
\hline$x, y, \dot{x}, \dot{y}$ & Cartesian body position and velocities \\
$r, \theta, \dot{r}, \dot{\theta}$ & Leg length, leg angle and velocities \\
$\tau$ & Hip torque command during stance \\
$y_{a}, \dot{x}_{a}$ & Apex height and velocity \\
$\theta_{t d}, \dot{r}_{t d}, \dot{\theta}_{t d}$ & Touchdown leg angle, polar velocities \\
$t_{b}, r_{b}, \theta_{b}$ & Bottom time, leg length and angle \\
$t_{l o}, r_{l o}, \theta_{l o}, \dot{r}_{l o}, \dot{\theta}_{l o}$ & Liftoff time, leg length, angle and velocities \\
$p_{\theta}$ & Angular momentum around the toe \\
\hline \hline \multicolumn{2}{|c|}{ Kinematic and Dynamic Parameters } \\
\hline$m, g$ & Body mass and gravitational acceleration \\
$k, r_{0}, c$ & Leg stiffness, rest length and damping \\
\hline
\end{tabular}

During flight, the body obeys ballistic flight dynamics

$$
\left[\begin{array}{l}
\ddot{x} \\
\ddot{y}
\end{array}\right]=\left[\begin{array}{c}
0 \\
-g
\end{array}\right]
$$

and the massless leg can be arbitrarily positioned. In contrast, during stance, the toe remains stationary on the ground while the body mass feels forces generated by both the passive spring-damper pair and the hip torque. The stance dynamics of the planar SLIP model in polar leg coordinates with respect to the toe location take the form

$$
\frac{d}{d t}\left[\begin{array}{c}
m \dot{r} \\
m r^{2} \dot{\theta}
\end{array}\right]=\left[\begin{array}{c}
m r \dot{\theta}^{2}-m g \cos \theta-k\left(r-r_{0}\right)-c \dot{r} \\
m g r \sin \theta+\tau
\end{array}\right] .
$$

A very useful abstraction for the analysis and control of cyclic TD-SLIP trajectories is provided by the apex return map, defined as a Poincaré map from one apex point to the next. In the following sections, we will use this map to study stability properties of TD-SLIP, and later adopt it as a tasklevel gait representation for a closed-loop running controller.

The apex return map can be formulated as $P:=P_{a} \circ P_{s} \circ P_{d}$ by composing three individual submaps $P_{d}, P_{s}, P_{a}$ for the descent, stance and ascent phases, respectively. The descent and ascent maps are trivial and are given by

$$
\begin{aligned}
& P_{d}:\left[\begin{array}{c}
\dot{r}_{t d} \\
r_{0} \dot{\theta}_{t d}
\end{array}\right]=R\left(\pi / 2-\theta_{t d}\right)\left[\begin{array}{c}
-\dot{x}_{a} \\
\sqrt{2 g\left(y_{a}-r_{0} \cos \theta_{t d}\right)}
\end{array}\right] \\
& P_{a}:\left[\begin{array}{c}
y_{a} \\
\dot{x}_{a}
\end{array}\right]=\left[\begin{array}{c}
r_{l o} \cos \theta_{l o}+\dot{y}_{l o}^{2} /(2 g) \\
\dot{x}_{l o}
\end{array}\right]
\end{aligned}
$$


where $\dot{x}_{l o}$ and $\dot{y}_{l o}$ are liftoff velocities in Cartesian coordinates and $R$ denotes the standard 2D rotation matrix. Unfortunately, the dynamics of (1) are not integrable in closed form. Consequently, we will use an analytical approximation for the stance map, which we describe in the next section.

\section{B. An Approximate Stance Map for the Unforced TD-SLIP}

A new analytical approximation to the dynamics of a dissipative SLIP model was proposed in [4]. However, this method assumes the presence of radial leg actuation, either in the form of a controllable leg stiffness, or the regulation of touchdown and liftoff leg lengths. In this section, we briefly review their method and extend it to support the hip torque actuation of our model.

The approximation proposed in [4], which, in turn, is based on the methods described in [16], relies on two key assumptions: 1). The angular travel throughout stance is relatively small and remains close to the vertical, allowing linearization of the gravitational potential in the Lagrangian with subsequent conservation of the angular momentum $p_{\theta}:=m r^{2} \dot{\theta}$ and 2). the radial compression is small with $r_{0}-r \ll r_{0}$, allowing a truncated Taylor expansion of related terms.

As described in [4], under these conditions and assuming, for now, that $\tau=0$, the radial component of (1) reduces to

$$
\ddot{r}+(c / m) \dot{r}+\left(\omega_{0}^{2}+3 \omega^{2}\right) r=-g+r_{0} \omega_{0}^{2}+4 r_{0} \omega^{2},
$$

where we define $\omega_{0}:=\sqrt{k / m}$ and $\omega:=p_{\theta} /\left(m r_{0}^{2}\right)$. Solutions to this simple second-order ODE can be found as

$$
r(t)=e^{-\zeta \hat{\omega}_{0} t}\left(A \cos \left(\omega_{d} t\right)+B \sin \left(\omega_{d} t\right)\right)+F / \hat{\omega}_{0}^{2},
$$

where we have $\hat{\omega}_{0}:=\sqrt{\omega_{0}^{2}+3 \omega^{2}}, \zeta:=c /\left(2 m \hat{\omega}_{0}\right), \omega_{d}:=$ $\hat{\omega}_{0} \sqrt{1-\zeta^{2}}, F:=-g+r_{0} \omega_{0}^{2}+4 r_{0} \omega^{2}$ and $A$ and $B$ determined by touchdown states as

$$
\begin{aligned}
A & :=r_{0}-F / \hat{\omega}_{0}^{2}, \\
B & :=\left(\dot{r}_{t d}+\zeta \hat{\omega}_{0} A\right) / \omega_{d} .
\end{aligned}
$$

Simple differentiation and further simplification yields radial TD-SLIP trajectories as

$$
\begin{aligned}
& r(t)=M e^{-\zeta \hat{\omega}_{0} t} \cos \left(\omega_{d} t+\phi\right)+F / \hat{\omega}_{0}^{2}, \\
& \dot{r}(t)=-M \hat{\omega}_{0} e^{-\zeta \hat{\omega}_{0} t} \cos \left(\omega_{d} t+\phi+\phi_{2}\right),
\end{aligned}
$$

with $M, \phi$ and $\phi_{2}$ determined through trigonometric identities. At this point, the angular trajectories can be determined using the constant angular momentum. An additional linearization of the term $1 / r^{2}$ leads to an analytical solution for the rate of change of the leg angle as

$$
\begin{aligned}
\dot{\theta}(t)= & 3 \omega-2 \omega F /\left(r_{0} \hat{\omega}_{0}^{2}\right)- \\
& 2 \omega M e^{-\zeta \hat{\omega}_{0} t} \cos \left(\omega_{d} t+\phi\right) / r_{0},
\end{aligned}
$$

integrated to yield the angular trajectory

$$
\begin{aligned}
\theta(t)= & \theta_{t d}+X t+ \\
& Y\left(e^{-\zeta \hat{\omega}_{0} t} \cos \left(\omega_{d} t+\phi+\phi_{3}\right)-\cos \left(\phi+\phi_{3}\right)\right) .
\end{aligned}
$$

with $X, Y$ and $\phi_{3}$ computed accordingly as in [4].
The final step in completing the stance map requires finding the time of liftoff. Only one of the two liftoff conditions described in [4] is applicable in the context of the present paper since we do not allow control of the liftoff leg length. Consequently, the liftoff time is solely determined by the solution to the equation $k\left(r_{0}-r\left(t_{l o}\right)\right)-c \dot{r}\left(t_{l o}\right)=0$, for which a sufficiently accurate analytical approximation can be found by approximating the exponential coefficient in the radial solution of (6) by its value at a specific instant during decompression. In particular, noting that the compression and decompression times are roughly equal, we use $e^{-\zeta \hat{\omega}_{0} t} \approx$ $e^{-\zeta \hat{\omega}_{0} 2 t_{b}}$, where $t_{b}$ denotes the bottom time, easily found by solving (7). Under this assumption, we have

$$
\begin{aligned}
t_{l o} & \approx\left(2 \pi-\arccos \left(k\left(r_{0}-F / \hat{\omega}_{0}^{2}\right) /\left(\bar{M} M e^{-\zeta \hat{\omega}_{0} \gamma t_{b}}\right)\right)\right. \\
& \left.-\phi-\phi_{4}\right) / \omega_{d},
\end{aligned}
$$

which yields the stance map as

$$
P_{s}:\left[\begin{array}{c}
r_{l o} \\
\theta_{l o} \\
\dot{r}_{l o} \\
\dot{\theta}_{l o}
\end{array}\right]=\left[\begin{array}{c}
r\left(t_{l o}\right) \\
\theta\left(t_{l o}\right) \\
\dot{r}\left(t_{l o}\right) \\
\dot{\theta}\left(t_{l o}\right)
\end{array}\right] .
$$

where the right hand side is a function of touchdown states.

Note, however, that these derivations completely ignore the presence of the hip torque. In the next section, we propose a new method to incorporate the effects of the hip torque through a fixed correction on the angular momentum value $p_{\theta}$ in a way similar to the one used in [5] for gravity corrections.

\section{Stance Map for the Torque Controlled TD-SLIP}

Hip actuation in legged systems can serve a number of different purposes. Among both biological [1] and robotic [13, $19,27]$ systems, its most common uses involve retraction of legs in flight and control of body posture with legs in stance. Interestingly, the use of hip actuation to provide thrust has not been studied as extensively in the robotics literature. In addition to a few direct experimental inquiries $[12,32]$ and indirect uses in multi-legged platforms [26,30], it has received limited attention in [2] in the form of an active spring.

In the present paper, we propose an open-loop hip actuation regime that enforces the ramp torque profile

$$
\tau(t)= \begin{cases}\tau_{0}\left(1-\frac{t}{t_{f}}\right) & \text { if } 0 \leq t \leq t_{f} \\ 0 & \text { if } t>t_{f}\end{cases}
$$

during stance, with $\tau_{0}$ and $t_{f}$ chosen prior to touchdown. This open-loop profile has three important advantages. Firstly, its simple functional dependence on time allows us to easily incorporate its effects into the derivations of the previous section. Second, if we choose $t_{f}$ to be the liftoff time, we have $\tau\left(t_{l o}\right)=0$, which prevents premature leg liftoff due to the action of the hip and ensures a structural match to the trajectories of the unforced system. Finally, its unidirectional action ensures that no negative work is done during stance.

Inspection of the TD-SLIP dynamics of (1) shows that the hip torque directly acts on the angular dynamics and only indirectly effects radial motion. Consequently, we hypothesize 
that an average correction to the constant angular momentum $p_{\theta}$ of Section II-B can capture the effects of the hip torque on system trajectories. Normally, the angular momentum during stance can be formulated as

$$
p_{\theta}(t)=p_{\theta}(0)+\int_{0}^{t} \tau(\eta) d \eta+\int_{0}^{t} m g r(\eta) \sin \theta(\eta) d \eta,
$$

by integrating the angular dynamics. Adopting the method proposed in [5], we compute a corrected angular momentum

$$
\hat{p_{\theta}}=p_{\theta}(0)+\Delta p_{\tau}+\Delta p_{g} .
$$

where $\Delta p_{\tau}$ and $\Delta p_{g}$ incorporate the time averaged effects of the leg torque and gravitational acceleration, respectively. Assuming $t_{f}=t_{l o}$, we have

$$
\Delta p_{\tau}:=\frac{1}{t_{l o}} \int_{0}^{t_{l o}}\left(\int_{0}^{\eta_{1}} \tau\left(\eta_{2}\right) d \eta_{2}\right) d \eta_{1}=\tau_{0} \frac{t_{l o}}{3} .
$$

However, even with available analytic approximations, derivation of an exact closed-form expression for $\Delta p_{g}$ is not feasible. Instead, we use a linear approximation to the integrand $r(\eta) \sin \theta(\eta)$ using its values at the touchdown and liftoff, resulting in

$$
\Delta p_{g}:=\frac{m g t_{l o}}{6}\left(2 r_{0} \sin \theta_{t d}+r_{l o} \sin \theta_{l o}\right) .
$$

Estimated values for the liftoff time $t_{l o}$, leg angle $\theta_{l o}$ and leg length $r_{l o}$ are provided by the unforced approximations of the previous section. Substituting $\hat{p_{\theta}}$ for the constant angular momentum in all derivations of Section II-B, we obtain a new approximation that takes into account the effects of both the hip torque and gravity on the stance trajectories.

Note that the corrections we propose have an iterative character since both (15) and (16) use prior estimates of $t_{l o}$ and $\theta_{l o}$. Consequently, starting from the unforced approximations, it is possible to iteratively apply these corrections to obtain more accurate predictions at the expense of analytic simplicity. Our simulations show that more than a single iteration is only needed for extreme conditions such as the angle of attack being very close to the touchdown leg angle, causing a bounce-back.

\section{Stability of AN ENERGY-Regulated TD-SLIP}

\section{A. Compensation of Damping Losses}

Unlike previous stability studies of lossless spring-mass hoppers, fully passive self-stabilization with a fixed touchdown angle and no active control is not possible with the TDSLIP model since damping losses will eventually drain out all energy in the system. Consequently, active hip thrust must be employed to sustain locomotion.

Recall that our choice of the hip torque in (12) incorporates two parameters: $\tau_{0}$ and $t_{f}$. We have already shown that choosing $t_{f}=t_{l o}$ is advantageous in preventing early liftoff and ensuring structural correspondence of system trajectories to our analytical approximation. The simplest possible strategy for the remaining parameter $\tau_{0}$, very close in spirit to the radial actuation strategy adopted by Raibert's runners [27] and its subsequent analysis in [21], is to choose a particular value and keep it constant across subsequent strides. The underlying idea is that since damping losses are proportional to the total energy level of the system, constant energy input will give rise to trajectories at a stable energy level. Unfortunately, in our 2-DOF model, such a strategy results in a two dimensional return map, for which, analytical solution and characterization of equilibrium points is not feasible.

Another possibility, which we adopt in the present paper, is to use the hip torque to compensate for all dissipative effects within a single step, ensuring conservation of energy in the apex return map and hence reducing its dimension by one. Note that the total energy dissipated within a single TD-SLIP step is given by

$$
E_{\text {loss }}=E_{c}+E_{k},
$$

where $E_{c}$ represents damping losses with

$$
E_{c}:=\int_{0}^{t_{l o}} c \dot{r}^{2}(\eta) d \eta
$$

and $E_{k}:=\left(r_{l o}-r_{0}\right)^{2} / 2$ captures the leftover energy in the leg spring when it lifts off before it is fully extended due to damping. Fortunately, our analytic approximations provide closed form expressions for both of these components. In particular, damping losses can be approximately computed as

$$
\begin{aligned}
E_{c} & =\frac{-c / M^{2} \hat{\omega}_{0}}{4 \zeta}\left(\zeta \cos \left(2\left(\phi+\phi_{2}\right)+\phi_{3}\right)+1\right. \\
& \left.-e^{-2 \zeta \hat{\omega}_{0} t_{l o}}\left(\zeta \cos \left(2 \omega_{d} t_{l o}+2\left(\phi+\phi_{2}\right)+\phi_{3}\right)+1\right)\right),
\end{aligned}
$$

while $E_{k}$ only depends on the previously computed $r_{l o}$.

In contrast, the energy supplied by the hip torque is

$$
E_{\tau}=\tau_{0} \int_{0}^{t_{l o}}\left(1-\frac{t}{t_{l o}}\right) \dot{\theta}(t) d t
$$

for which our analytical approximations can also be used to obtain closed-form expressions. We omit the details here for space considerations. Since both (17) and (20) can be obtained in closed form as a function of initial conditions and the choice of touchdown angle $\theta_{t d}$, we can easily find the desired torque magnitude $\tau_{0}$ by solving

$$
E_{\tau}=E_{\text {loss }}
$$

As noted above, this choice of torque results in successive apex states having the same energy, at least while working within our approximate apex return map. Naturally, additional corrections would be needed to apply these ideas to the exact plant model since inaccuracies of our approximations would invalidate this conservation. Nevertheless, we use this active compensation regime to reduce the dimension of our analytic apex return map, allowing us to easily identify its equilibrium points and characterize their stability.

\section{B. Equilibrium Points with a Fixed Leg Placement Policy}

In this section, we use our analytic approximations to identify and characterize equilibrium points of the one dimensional "energy-regulated" return map on the apex height $y_{a}$ arising from the use of a fixed touchdown angle policy with 
$\theta_{t d}=\beta$ and the energy-regulating hip torque described in Section III-A. All results in this section will be presented in non-dimensional versions of relevant variables, defined as

$$
\begin{aligned}
\bar{y}_{a} & :=y_{a} / r_{0} \\
\dot{\bar{x}}_{a} & :=\dot{x}_{a} / \sqrt{g r_{0}} \\
\bar{E}_{a} & :=E_{a} /\left(m g r_{0}\right) \\
\bar{k} & :=k r_{0} /(m g) \\
\zeta_{0} & :=c /(2 \sqrt{m k}) .
\end{aligned}
$$

Finally, in order to facilitate comparison with earlier studies, we use kinematic and dynamic parameters that roughly match those of an average human with $m=80 \mathrm{~kg}$ and $r_{0}=1 \mathrm{~m}$.

Fig. 3 shows two families of return maps for $\beta=20^{\circ}$ and $\beta=32^{\circ}$, respectively, together with the dependence of equilibrium points on the energy level of the system. These results show that the TD-SLIP exhibits asymptotically stable behavior under the fixed touchdown angle, energy-regulated regime, with the location of the equilibrium point depending on the chosen energy level. We can also observe that as the fixed touchdown angle $\beta$ increases, the energy range for which stable fixed points exist increases as well. This is rather natural since the torque actuation at the hip can only supply energy through the angular momentum, which directly increases the angular span during stance. Increasing the touchdown angle admits a larger angular span for stance, allowing stable fixed points to form at higher energy levels as well.
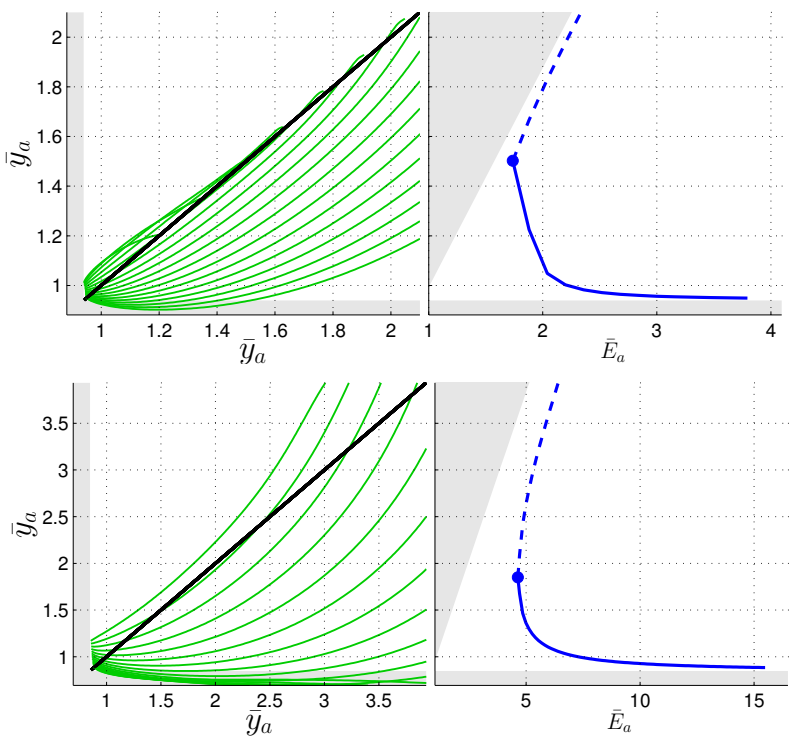

Fig. 3. Apex height return map (left) and associated equilibrium points (right) for the TD-SLIP model as a function of different (dimensionless) energy levels, generated with the proposed analytical approximations. The top plots are obtained with $\beta=20^{\circ}, k=40, \zeta_{0}=0.07$, whereas the bottom plots use $\beta=32^{\circ}, \bar{k}=40, \zeta_{0}=0.07$ in dimensionless coordinates. Solid and dashed lines in the right figure indicate stable and unstable equilibrium points respectively. Shaded regions correspond to kinematically infeasible configurations.

Having established the presence of stable equilibrium points for the torque-controlled SLIP model, Fig. 4 shows a comparison of fixed points predicted by our analytic approximations,
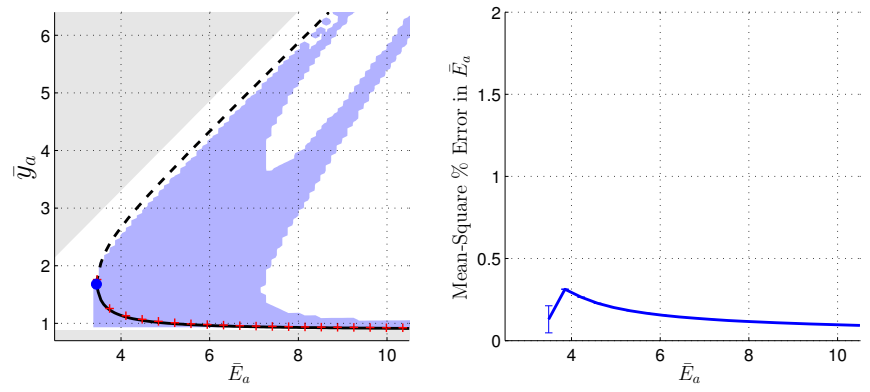

Fig. 4. Left: Comparison of stable equilibrium points predicted by our analytic approximation (solid line) with those obtained by numeric simulation of TD-SLIP dynamics (plus signs) for $\beta=28^{\circ}$ and different apex energy levels in the range $\bar{E}_{a} \in[2,10]$. Shaded region in the middle illustrates the stable domain of attraction for the simulated plant model. Right: Percentage mean-square error between initial and steady-state dimensionless energy levels for the simulated plant.

with those that arise within simulations of the exact TDSLIP model. In order to make direct comparisons possible, we started TD-SLIP simulations from a large range of initial $y_{a}$ and $E_{a}$ values, with a fixed touchdown angle and an energy regulation controller similar to the one presented Section IIIA, but now taking the energy level of the very first step as an overall regulation goal. This modification was necessary since using the approximations to locally enforce energy conservation at every step would slowly cause prediction errors to accumulate, either draining all energy out of the system, or causing it to diverge. We then checked whether the system converges to a stable equilibrium point in apex coordinates after 25 steps up to a tolerance of $10^{-4}$. Shaded region in the middle of the left plot of Fig. 4 illustrates the resulting domain of attraction, while the plus marks in the same plot illustrate the associated set of fixed points.

Note, also, that the domain of attraction exhibited by the simulation almost exactly covers the region between the unstable and stable fixed points predicted by our approximations. There is also an almost exact match between the fixed points predicted by our approximations and those obtained from simulation. The cavities to the right of the region of attraction arise from the presence of the "gap" region in the return map, resulting from kinematic constraints that require the apex height to be sufficiently large to allow leg placement. The reason for this can be clearly seen in the bottom right plot of Fig. 3, where parts of the return map overlap with the kinematically infeasible gray region on the bottom. This means that some initial conditions at high energy levels will lead to apex states for which leg placement at an angle of $\beta$ is impossible. This gap was also observed by previous studies [16], and is reproduced by both our analytical approximations, and the simulated plant.

The right plot in Fig. 4 shows the mean and standard deviations of the percentage mean-square energy difference between the initial and steady state apex points for the simulated plant. The fact that this difference is consistently below $0.3 \%$ shows that our approximations are capable of very accurately modeling energy losses and successfully predict fixed points of 
the exact TD-SLIP plant. It is worth noting, also, that accuracy also increases significantly with increasing energy levels.

\section{Parameter Dependence of Equilibrium Points}

Equilibrium points that arise from our fixed touchdown angle, energy-regulated regime naturally depend on the kinematic and dynamic parameter choices. Fig. 5 illustrates the dependence of stable fixed points on each individual parameter (the touchdown angle $\beta$, the dimensionless leg stiffness $\bar{k}$ or leg damping $\zeta_{0}$ ) with the remaining two parameters kept constant. The leftmost figure mirrors our observations in the previous section, namely that the range of stable energy levels increase with larger touchdown angles.
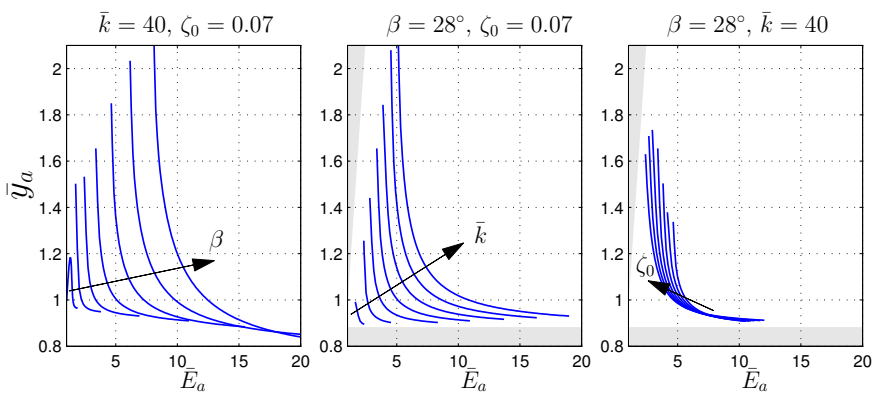

Fig. 5. Dependence of stable equilibrium points on variations of the touchdown angle $\beta$ (left), leg spring stiffness $\bar{k}$ (middle) and leg damping ratio $\zeta_{0}$ (right). Arrows indicate increasing directions for each varied parameter.

The dependence of equilibrium points on the leg stiffness, illustrated in the middle figure shows that increasing spring constants cause an increase in the range of stable energy levels. This is also natural since an increased stiffness corresponds to shorter stance times, resulting in decreased damping losses and a corresponding decrease in the necessary torque input. Finally, we observe that the impact of the damping coefficients on the equilibrium points is not as pronounced, providing evidence that our compensation strategy successfully balances damping losses. Nevertheless, increasing the amount of damping causes a slight decrease in the range of stable energy levels.

\section{Correspondence of the Model to Biological Data}

A recent quantitative comparison of ground reaction force data from a variety of running animals to those predicted by a simple, passive spring-mass model shows that despite the very good correspondence of vertical force components between biological data and the idealized SLIP model, there are some discrepancies in how well horizontal forces can be predicted [38]. In this section, we report on an interesting property of the torque-actuated TD-SLIP morphology: It seems to be capable of qualitatively reproducing ground reaction force profiles very similar to those observed in biological systems.

Fig. 6 illustrates the body trajectory for a single stride of steady-state running with the TD-SLIP model, together with a depiction of "virtual footfalls" in the direction of instantaneous ground reaction force vectors throughout the stance phase. As a result of the ramp torque profile we use for supplying energy to the system, large backward horizontal forces are

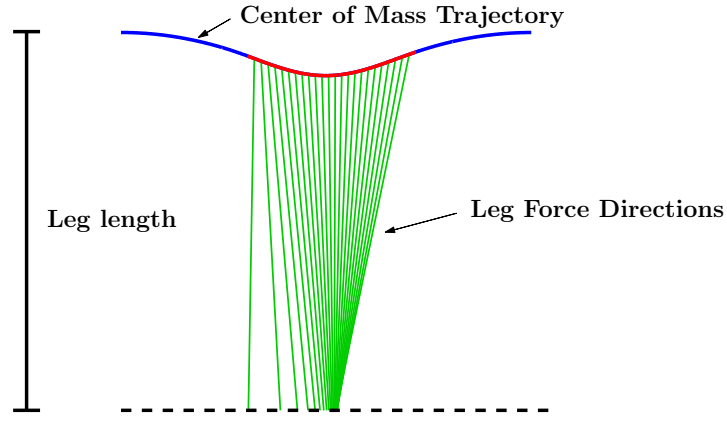

Fig. 6. COM trajectory for a single steady-state stride of the TD-SLIP model with $\bar{k}=40, \zeta_{0}=0.07$, running at approximately $3 \mathrm{~m} / \mathrm{s}(0.96$ in dimensionless units). Comparison of ground reaction force directions during stance to biological data presented in [38] reveals a remarkable qualitative match.

introduced in the beginning of the stance phase, resulting in associated virtual footfalls appearing behind the actual toe location. Towards the end of the stance phase, the hip torque approaches zero and brings the virtual footfall and actual toe locations together.

This qualitative structure is observed for all steady-state trajectories of the TD-SLIP model and is remarkably consistent with biological data presented in [38]. Even though we do not yet have any quantitative basis in which any predictive claims can be made, we think that this correspondence may provide evidence towards the use of hip torque as an additional source of energy used by biological runners, improving the predictive accuracy and utility of dynamic models of running.

\section{Feedback Control of TD-SLIP RunNing}

\section{A. Deadbeat Control by Inversion of the Apex Return Map}

The presence of a sufficiently accurate analytic formulation of the apex return map naturally motivates its inversion to obtain a controller for stabilizing the system around a desired operating point $\left[y_{a}^{*}, \dot{x}_{a}^{*}\right]$ in apex state coordinates. A similar approach was adopted in a number of studies $[8,29,31]$, but never in the context of a lossy model or torque actuation. In this section, we describe a deadbeat gait controller for TD-SLIP as an application of our approximations, and show that it is capable of very accurately regulating the apex states of a running TD-SLIP and improves on both the accuracy and stability of previous attempts to control a similar, torqueactuated model in [12].

An explicitly specified desired apex state will require a nonzero change in the energy level of the system. Using a strategy similar to the energy-conserving torque controller of Section III-A, we will use the hip torque to supply the requested energy input to the system in a single step. Similar to (21), this energy is given by

$$
E_{\tau}=\frac{1}{2} m\left(\left(\dot{x}_{a}^{*}\right)^{2}-\dot{x}_{a}^{2}\right)+m g\left(y_{a}^{*}-y_{a}\right)+E_{\text {loss }},
$$

which can easily be solved to determine the ramp torque magnitude $\tau_{0}$, assuming, once again, that $t_{f}=t_{l o}$. 
Once the desired torque profile is determined, the return map has only one remaining degree of control freedom: the touchdown angle $\theta_{t d}$. A deadbeat controller can be formulated as a one dimensional minimization problem in the form

$$
\theta_{t d}=\underset{\frac{-\pi}{2}<\theta<\frac{-\pi}{2}}{\operatorname{argmin}}\left(\dot{x}_{a}^{*}-\left(\pi_{\dot{x}_{a}} \circ P\left(\theta_{t d},\left[y_{a}, \dot{x}_{a}\right]_{k}\right)\right)\right)^{2},
$$

whose numerical solution is trivial due to the availability of our analytic approximation for the return map $P$. This yields an effective, one-step deadbeat controller for the regulation of forward speed and hopping height for the TD-SLIP model.

\section{B. Controller Performance and Comparison}

As noted before, there are very few in depth studies of how hip torque actuation can be used to achieve stable locomotion. Among notable exceptions is recent work on locomotion over mildly rough terrain [12] where the authors use TDSLIP equations of motion to derive an approximate energy controller to regulate hopping height, and a PD-based torque policy to regulate forward speed. In this section, we present a comparison of the new controller we described in Section IVA, with the control strategy proposed in [12]. In order to maintain consistency with our previous stability results, we use the same kinematic and dynamic parameters with Section III$\mathrm{B}$, roughly corresponding to an average human morphology. Note that parameters used in [12] are not substantially different from ours when converted to dimensionless units. All simulations were run in Matlab using a fourth order RungeKutta integrator together with accurate detection of transition events. Each run consisted of 25 steps, at the end of which we determined whether there was convergence to a fixed point in apex coordinates.

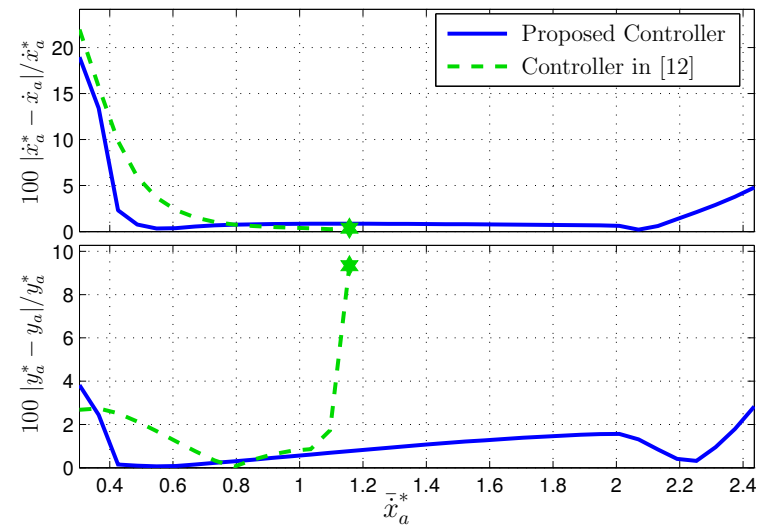

Fig. 7. Comparison of tracking performance for apex speed (top) and apex height (bottom) between the proposed controller (solid) and the PD control policy described in [12] (dashed) as a function of (dimensionless) desired velocity $\bar{x}_{a}^{*}$. Markers indicate where the controller of [12] loses stability. Vertical axes are percentage errors.

Fig. 7 illustrates tracking performances of both controllers for apex speed and height variables in terms of normalized percentage error measures. Note that our controller based on an accurate analytic model for the dynamics of TD-SLIP significantly increases the range of velocity goals that can be achieved without losing stability. Moreover, improvements can be observed in the tracking accuracy for both the apex speed and height variables. Finally, our controller does not require any feedback or sensory measurements during stance, but relies only on accurate measurement of apex states. This makes practical implementations much more feasible compared to the active PD control strategy since high-bandwidth feedback is usually very challenging for fast legged robots.

\section{COnclusions And Future Work}

In this paper, we presented a novel method to obtain analytical approximations to the stance trajectories of a dissipative, torque actuated planar spring-mass hopper. We have successfully used our approximations to design an energyregulation controller for the hip torque that can accurately compensate for the effects of damping within a stride, allowing us to obtain a one-dimensional return map under a fixed angle leg placement policy. Consequently, we were able to analyze stability properties of this model, identifying and characterizing its equilibrium points. The predictive accuracy of our analytical approximations was confirmed by a very close match to fixed points and their domains of attraction obtained through numerical simulations of the exact plant model. We have also demonstrated the utility of our approximations through their use in designing a gait controller.

It is important to note that neither the energy regulating hip torque controller, nor the subsequent stability analysis would have been possible in the absence of our analytical approximations. Consequently, we believe this paper presents the first careful study of stability properties of running in the presence of non-negligible damping. In this context, we believe that the incorporation of damping as a significant component in the dynamical model substantially increases the applicability of associated analytical tools and controllers to practical robot platforms in which dissipative effects will always be present and may sometimes be a dominant factor particularly if compliance is achieved through composite materials. In the future, we hope to demonstrate the practical utility of our approximations by experimental verification of their predictions with respect to a physical monopedal runner.

Our choice of hip torque as the primary source of energy input to the system was motivated by the difficulty of implementing radial actuation in physical robot platforms, and the simplicity and success of existing robot platforms with similar actuation mechanisms $[26,30]$. We have also further simplified our model by assuming a fixed body angle that may be justified by morphologies in which additional legs on the front and back of the body provide a stabilizing effect, or where the body link is explicitly constrained by an experimental setup $[12,32]$. In this context, we discovered an interesting correspondence between the ground reaction force profiles resulting from the use of a hip torque and biological data presented in [38], leading to a possible explanation for the inability of the original SLIP model in reproducing horizontal force components during running and a very preliminary hypothesis that hip torque may be playing a previously unaddressed important role 
in the control of legged locomotion. In the future, we hope to generalize our results to a freely rotating body link, making the results applicable to less constrained morphologies such as bipeds. For example, one of the interesting possibilities is how forward-bending body posture and the resulting gravitational torque can be used to balance the torque input from the hip, making it possible to both have a freely rotating body, while using the hip torque to provide thrust. This seems to be one of the ways in which ideas similar to those used for passive dynamic walking can be applied to efficient bipedal running and we hope to extend our results in this paper to such scenarios.

\section{ACKNOWLEDGMENTS}

M. Mert Ankaralı was partially supported by the National Scientific and Technological Research Council of Turkey (TUBITAK). This project was also partially supported by TUBITAK project 109E032. Finally, we thank Afsar Saranli for inspiring discussions and his support for this work.

\section{REFERENCES}

[1] R. Alexander. Principles of Legged Locomotion. Princeton University Press, 2006.

[2] R. Altendorfer, D. E. Koditschek, and P. Holmes. Stability Analysis of a Clock-Driven Rigid-Body SLIP Model for RHex. Int. Journal of Robotics Research, 23(10-11):1001-1012, 2004.

[3] R. Altendorfer, D. E. Koditschek, and P. Holmes. Stability Analysis of Legged Locomotion Models by Symmetry-Factored Return Maps. Int. Journal of Robotics Research, 23(10-11):979-999, 2004.

[4] M. M. Ankarali, O. Arslan, and U. Saranli. An analytical solution to the stance dynamics of passive spring-loaded inverted pendulum with damping. In 12th Int. Conf. on Climbing and Walking Robots and The Support Technologies for Mobile Machines (CLAWAR'09), Istanbul, Turkey, September 2009.

[5] O. Arslan, U. Saranli, and O. Morgul. An aproximate stance map of the spring mass hopper with gravity correction for nonsymmetric locomotions. In Proc. of the IEEE Int. Conf. on Robotics and Automation, Kobe, Japan, May 2009.

[6] R. Blickhan. The spring-mass model for running and hopping. J. of Biomechanics, 22:1217-1227, 1989.

[7] R. Blickhan and R. J. Full. Similarity in multilegged locomotion: Bouncing like a monopode. J. of Comparative Physiology A: Neuroethology, Sensory, Neural, and Behavioral Physiology, 173(5):509517, Nov. 1993.

[8] S. G. Carver, N. J. Cowan, and J. M. Guckenheimer. Lateral stability of the spring-mass hopper suggests a two-step control strategy for running. Chaos: An Interdisciplinary Journal of Nonlinear Science, 19(2):026106, 2009

[9] G. A. Cavagna, F. P. Saibene, and R. Margaria. Mechanical work in running. J. of Applied Physiology, 19(2):249-256, 1964.

[10] J. G. Cham, S. A. Bailey, J. E. Clark, R. J. Full, and M. R. Cutkosky. Fast and robust: Hexapedal robots via shape deposition manufacturing. Int. Journal of Robotics Research, 21(10):869-882, 2002.

[11] N. Cherouvim and E. Papadopoulos. Speed and height control for a special class of running quadruped robots. In Proceedings of the IEEE Int. Conf. on Robotics and Automation, pages 825-830, May 2008.

[12] N. Cherouvim and E. Papadopoulos. Control of hopping speed and height over unkown rough terrain using a single actuator. In Proceedings of the IEEE Int. Conf. on Robotics and Automation, Kobe, Japan, 2009.

[13] C. Chevallereau, E. R. Westervelt, and J. W. Grizzle. Asymptotically Stable Running for a Five-Link, Four-Actuator, Planar B ipedal Robot. Int. Journal of Robotics Research, 24(6):431-464, 2005.

[14] S. Collins, A. Ruina, R. Tedrake, and M. Wisse. Efficient Bipedal Robots Based on Passive-Dynamic Walkers. Science, 307(5712):1082-1085, 2005.

[15] M. Garcia, A. Chatterjee, A. Ruina, and M. Coleman. The simplest walking model: Stability, complexity, and scaling. J. of Biomechanical Engineering, 120(2):281-288, 1998.
[16] H. Geyer, A. Seyfarth, and R. Blickhan. Spring-mass running: simple approximate solution and application to gait stability. J. of Theoretical Biology, 232(3):315-328, Feb. 2005.

[17] H. Geyer, A. Seyfarth, and R. Blickhan. Compliant leg behaviour explains basic dynamics of walking and running. Proceedings of the Royal Society B: Biological Sciences, 273(1603):2861-2867, 2006.

[18] R. M. Ghigliazza, R. Altendorfer, P. Holmes, and D. Koditschek. A simply stabilized running model. SIAM Journal on Applied Dynamical Systems, 2:187-218, 2003.

[19] P. Gregorio, M. Ahmadi, and M. Buehler. Design, control, and energetics of an electrically actuated legged robot. Transactions on Systems, Man, and Cybernetics, 27(4):626-634, August 1997.

[20] P. Holmes, R. Full, D. Koditschek, and J. Guckenheimer. The dynamics of legged locomotion: Models, analyses, and challenges. SIAM Review, 48(2):207-304, 2006

[21] D. E. Koditschek and M. Buehler. Analysis of a simplified hopping robot. Int. Journal of Robotics Research, 10(6):587-605, 1991.

[22] A. D. Kuo. Stabilization of lateral motion in passive dynamic walking. Int. Journal of Robotics Research, 18(9):917-930, September 1999

[23] T. McGeer. Passive dynamic walking. Int. Journal of Robotics Research, 9(2):62-82, 1990

[24] T. A. McMahon and G. C. Cheng. The mechanics of running: How does stiffness couple with speed. J. of Biomechanics, 23(Suppl. 1):6578, 1990.

[25] R. Playter, M. Buehler, and M. Raibert. BigDog. In Society of PhotoOptical Instrumentation Engineers (SPIE) Conference Series, volume 6230 of Presented at the Society of Photo-Optical Instrumentation Engineers (SPIE) Conference, June 2006.

[26] I. Poulakakis, J. A. Smith, and M. Buehler. Modeling and Experiments of Untethered Quadrupedal Running with a Bounding Gait: The Scout II Robot. Int. Journal of Robotics Research, 24(4):239-256, 2005.

[27] M. Raibert. Legged robots that balance. MIT Press series in artificial intelligence. MIT Press, Boston, 1986.

[28] J. J. Robilliard and A. M. Wilson. Prediction of kinetics and kinematics of running animals using an analytical approximation to the planar spring-mass system. J Exp Biol, 208(23):4377-4389, 2005.

[29] U. Saranli. Dynamic Locomotion with a Hexapod Robot. PhD thesis, The University of Michigan, Ann Arbor, MI, September 2002.

[30] U. Saranli, M. Buehler, and D. E. Koditschek. RHex: A simple and highly mobile robot. Int. Journal of Robotics Research, 20(7):616-631, July 2001.

[31] U. Saranli, W. J. Schwind, and D. E. Koditschek. Toward the control of a multi-jointed, monoped runner. In Proc. of the IEEE Int. Conf. On Robotics and Automation, volume 3, pages 2676-82, New York, 1998.

[32] A. Sato and M. Buehler. A planar hopping robot with one actuator: design, simulation, and experimental results. In Proceedings of the IEEE/RSJ Int. Conf. on Intelligent Robots and Systems, volume 4, pages 3540-3545, Sept.-2 Oct. 2004

[33] J. Schmitt. A simple stabilizing control for sagittal plane locomotion. J. of Computational and Nonlinear Dynamics, 1(4):348-357, 2006.

[34] W. J. Schwind. Spring Loaded Inverted Pendulum Running: A Plant Model. Phd, University of Michigan, 1998.

[35] W. J. Schwind and D. E. Koditschek. Approximating the stance map of a 2-dof monoped runner. J. of Nonlinear Science, 10:533-568, 2000.

[36] A. Seyfarth, H. Geyer, M. Gunther, and R. Blickhan. A movement criterion for running. J. of Biomechanics, 35(5):649-655, May 2002.

[37] M. J. Spenko, G. C. Haynes, J. A. Saunders, M. R. Cutkosky, A. A. Rizzi, R. J. Full, and D. E. Koditschek. Biologically inspired climbing with a hexapedal robot. J. of Field Robotics, 25(4-5):223-242, 2008.

[38] M. Srinivasan and P. Holmes. How well can spring-mass-like telescoping leg models fit multi-pedal sagittal-plane locomotion data? J. of Theoretical Biology, 255(1):1 - 7, 2008.

[39] M. Vukobratovic and B. Borovac. Zero-moment point-thirty five years of its life. Int. Journal of Humanoid Robotics, 1(1):157-173, 2004.

[40] E. Westervelt, J. Grizzle, and D. Koditschek. Hybrid zero dynamics of planar biped walkers. IEEE Transactions on Automatic Control, 48(1):42-56, January 2003. 